\title{
Comparison of GEANT4 Simulations with Experimental Data for Thick Al Absorbers
}

\author{
Olga Yevseyeva ${ }^{a}$, Joaquim de Assis ${ }^{a}$, Ivan Evseev ${ }^{\mathrm{b}}$, Hugo Schelin ${ }^{\mathrm{b}}$, Sergei \\ Paschuk $^{\mathrm{b}}$, Edney Milhoretto ${ }^{\mathrm{b}}$, João Setti ${ }^{\mathrm{b}}$, Katherin Díaz ${ }^{\mathrm{c}}$, Joel Hormaza ${ }^{\mathrm{d}}$, \\ and Ricardo Lopes ${ }^{\mathrm{e}}$
}

\begin{abstract}
${ }^{a}$ Instituto Politécnico da UERJ, Rua Alberto Rangel sin ${ }^{\circ}, 28630-050$, Nova Friburgo-RJ, BRAZIL ${ }^{b}$ Universidade Tecnológica Federal do Paraná - UTFPR, Av. Sete de Setembro 3166, 80230-901, Curitiba-PR, BRAZIL

${ }^{c}$ Centro de Aplicaciones Tecnologicas y Desarrollo Nuclear-CEADEN, Calle $30 \$ 502$ e/5ta y $7 \mathrm{ma}$ Avenida, Playa, Ciudad Habana, CUBA

${ }^{d}$ Instituto de Biociências da UNESP, Distrito de Rubião Jr. s/n ${ }^{\circ}, 18618-000$, Botucatu - SP, BRAZIL ${ }^{e}$ Laboratório de Instrumentação Nuclear, COPPE UFRJ, Av. Horácio Macedo 2030, 21941-914, Rio de Janeiro - RJ BRAZIL
\end{abstract}

\begin{abstract}
Proton beams in medical applications deal with relatively thick targets like the human head or trunk. Therefore, relatively small differences in the total proton stopping power given, for example, by the different models provided by GEANT4 can lead to significant disagreements in the final proton energy spectra when integrated along lengthy proton trajectories. This work presents proton energy spectra obtained by GEANT4.8.2 simulations using ICRU49, Ziegler1985 and Ziegler2000 models for $19.68 \mathrm{MeV}$ protons passing through a number of $\mathrm{Al}$ absorbers with various thicknesses. The spectra were compared with the experimental data, with TRIM/SRIM2008 and MCNPX2.4.0 simulations, and with the Payne analytical solution for the transport equation in the Fokker-Plank approximation. It is shown that the MCNPX simulations reasonably reproduce well all experimental spectra. For the relatively thin targets all the methods give practically identical results but this is not the same for the thick absorbers. It should be noted that all the spectra were measured at the proton energies significantly above $2 \mathrm{MeV}$, i.e., in the so-called "Bethe-Bloch region". Therefore the observed disagreements in GEANT4 results, simulated with different models, are somewhat unexpected. Further studies are necessary for better understanding and definitive conclusions.
\end{abstract}

Keywords: Proton beams, Energy measurement, Monte Carlo methods.

PACS: $24.10 . \mathrm{Lx} ; 02.50 . \mathrm{Ng} ; 02.70 . \mathrm{Uu} ; 05.60 . \mathrm{Cd} ; 07.05 . \mathrm{Tp} ; 81.70 . \mathrm{Tx} ; 82.20 . \mathrm{Fd} ; 87.55 . \mathrm{km}$.

\section{INTRODUCTION}

The GEANT4 [1,2] simulations are essential for the development of complex systems like particle detectors for LHC at CERN, or proton computerized tomography (pCT) for medical applications [3-8]. In the last case, however, a proton beam passes trough relatively thick targets like the human head or trunk. Although GEANT4 has been validated by the USA National Institute of Standards and Technology - NIST PSTAR stopping powers (SP) for protons [9], the small differences in SP given by the different models provided by GEANT4 may lead to significant disagreements in the final proton energy spectra when integrated along proton trajectories. 
At least, our previous simulations [10-14] have shown that there are notable differences between the spectra, simulated by GEANT4 (in the standard execution mode) and the results of simulation by TRIM/SRIM2003 [15] and MCNPX [16]. These differences in the mean final proton energy are observed for $\mathrm{H}_{2} \mathrm{O}$ and $\mathrm{Al}$ absorbers at any thickness, but for relatively thin (less than $\sim 50 \%$ of the full range of protons with the same energy in the same material) targets are less than it could be resolved in a real experiment. For the thickest targets, however, these differences overcame the typical energy resolution.

In addition, it has been observed that there is an obvious scale effect: all the spectra simulated for the pairs of initial proton energies $25 \mathrm{MeV}-250 \mathrm{MeV}$ [11-13], and $23 \mathrm{MeV}-230 \mathrm{MeV}$ [14], could be scaled one to the other by applying a factor 10 to the energy scale. It should be noted that the spectra were simulated for target thicknesses equal to $10 \%$ to $90 \%$ of the corresponded full projected range from NIST PSTAR. Consequently, the $\sim 0.5 \mathrm{MeV}$ difference in simulated spectra for the thickest $(90 \%)$ water target at initial energy $23 \mathrm{MeV}$, for example, corresponds to $\sim 5 \mathrm{MeV}$ difference at $230 \mathrm{MeV}$, etc.

This work presents proton energy spectra obtained by GEANT4.8.2 simulations using ICRU49, Ziegler1985 and Ziegler2000 models for $19.68 \mathrm{MeV}$ protons passing through $\mathrm{Al}$ absorbers with various thicknesses. The spectra were compared with the experimental data [17], with TRIM/SRIM2008 and MCNPX2.4.0 simulations, and with the Payne analytical solution for the Boltzmann transport equation in the FokkerPlank approximation [18].

\section{METHODOLOGY}

The experimental data used in this work as reference were taken from the paper by Tschalär and Maccabee [17], where the spectra of $19.68 \mathrm{MeV}$ protons after passing through $0.099,0.2675,0.398$ and $0.497 \mathrm{~g} / \mathrm{cm}^{2}$ of $\mathrm{Al}$ were published as well as for other Al absorbers thicknesses. These data were chosen since they contain the most complete set of experimental spectra for protons passing through the biggest variety of thicknesses of Al targets in relation to full absorption range (see Table 1).

The NIST PSTAR and SRIM2008 values for the mean final proton energy after passing through a layer were extracted from correspondent reference tables using a cubic spline interpolation. The CSDA values were calculated based on the same form of Bethe-Bloch equation as it had been published in $[10,11]$. The calculations of proton energy spectra using Payne's theory [18] were based at the equations which could be found in $[10,11]$ also.

MCNPX is a general-purpose Monte Carlo radiation transport code developed at Los Alamos National Laboratory [16]. We were interested in this code because its applications include, among others, proton therapy. The simulation was done using the MCNPX version 2.4.0.

TRIM is historically one of the first Monte Carlo codes for ion transportation, actually with the friendliest interface [15]. In this work, we used the TRIM code from the SRIM2008 version 04 package.

GEANT4 $[1,2]$ is actually the most general framework for handling the motion of particles through matter. Its ability to work with very complex geometry and tracking 
is essential to the pCT development [10-14]. The simulations were made with the version GEANT4.8.2 by adjusting the parameters for the Hadrontherapy toolkit, as it is discussed in [19]. The simulations were performed using ICRU49, Ziegler1985 and Ziegler2000 models from the Low Energy Extension Pack following the results and discussion at [9].

All the Monte Carlo simulations were done in the narrow pencil beam and wide detector approach. In order to make a correct comparison of the spectra with different number of simulated protons, all the spectra were reduced to the discrete Probability Density Functions (PDF).

The standard analysis of statistical errors was done. The values of mean energies extracted from all simulated spectra are shown in TABLE 1 with the same number of significant digits as the exact results in the first 4 rows. However, the margin of statistical error in these values should be understood to be one-half the value of the pre last significant place, i.e. $0.005 \mathrm{MeV}$.

As for the values in TABLE 1, extracted from experimental data [17], the margin of total error is estimated to be less than approximately $0.02-0.03 \mathrm{MeV}$.

\section{RESULTS AND DISCUSSION}

The mean protons energies from simulated spectra are presented at TABLE 1:

TABLE 1. Mean energies of $19.68 \mathrm{MeV}$ protons after $\mathrm{Al}$ absorbers (in $\mathrm{MeV}$ ).

\begin{tabular}{|c|c|c|c|c|}
\hline $\begin{array}{l}\text { Absorber Thicknesses } \\
\text { (part of PSTAR Projected Range, [\%]) }\end{array}$ & $\begin{array}{c}\mathbf{0 . 0 9 9} \mathbf{g} / \mathrm{cm}^{2} \\
(17.79 \%)\end{array}$ & $\begin{array}{c}\mathbf{0 . 2 6 7 5} \mathbf{g} / \mathbf{c m}^{\mathbf{2}} \\
(48.07 \%)\end{array}$ & $\begin{array}{c}\mathbf{0 . 3 9 8 g} / \mathbf{c m}^{2} \\
(71.52 \%)\end{array}$ & $\begin{array}{c}\mathbf{0 . 4 9 7} \mathbf{g} / \mathbf{c m}^{2} \\
(89.31 \%)\end{array}$ \\
\hline NIST PSTAR & 17.614 & 13.566 & 9.616 & 5.440 \\
\hline SRIM2008 & 17.622 & 13.574 & 9.583 & 5.347 \\
\hline CSDA & 17.603 & 13.522 & 9.515 & 5.198 \\
\hline Payne's Theory & 17.964 & 13.522 & 9.512 & 5.185 \\
\hline MCNPX 2.4.0 & 17.637 & 13.602 & 9.667 & 5.514 \\
\hline TRIM/SRIM2008 & 17.611 & 13.557 & 9.587 & 5.378 \\
\hline GEANT4.8.2 (ICRU49) & 17.619 & 13.522 & 9.558 & 5.297 \\
\hline GEANT4.8.2 (Ziegler1985) & 17.664 & 13.694 & 9.867 & 5.832 \\
\hline GEANT4.8.2 (Ziegler2000) & 17.606 & 13.482 & 9.468 & 5.113 \\
\hline Experiment & 17.632 & 13.602 & 9.722 & 5.558 \\
\hline
\end{tabular}

It is evident that in the case of thin, $0.099 \mathrm{~g} / \mathrm{cm}^{2}$ absorber all the results are very similar. That is why, to visualize the differences, only the spectra simulated for the thickest, $0.497 \mathrm{~g} / \mathrm{cm}^{2}$ target are shown in FIGURE 1.

As seen from the values from TABLE 1, however, there is a similarity in results obtained for all absorber thicknesses. The GEANT spectra, simulated with Zigler1985 model, are always more energetic than the experimental data. The MCNPX spectra are in the best agreement with the experiment. The TRIM spectra are also close to the experimental ones, but always have a little bit lower energy. The use of the ICRU49 model always leads to a less overestimation of proton energy loss than the Ziegler2000 model, but still gives the mean energies below the TRIM results. At the same time, the theory predicts mean proton energies between the GEANT results with these two models. It should be noted that a special correction procedure has been applied to the 
theoretical results in [17] to arrange the coincidence with experimental data. The results of present simulations are presented in Table 1 without any adjustment.

It should be also noted that all proton spectra were obtained at the energy region significantly above $0.5 \mathrm{MeV}$, i.e., in the so-called "Bethe-Bloch region" [9]. Therefore the observed disagreements in GEANT4 results, simulated with different models, are somewhat unexpected.

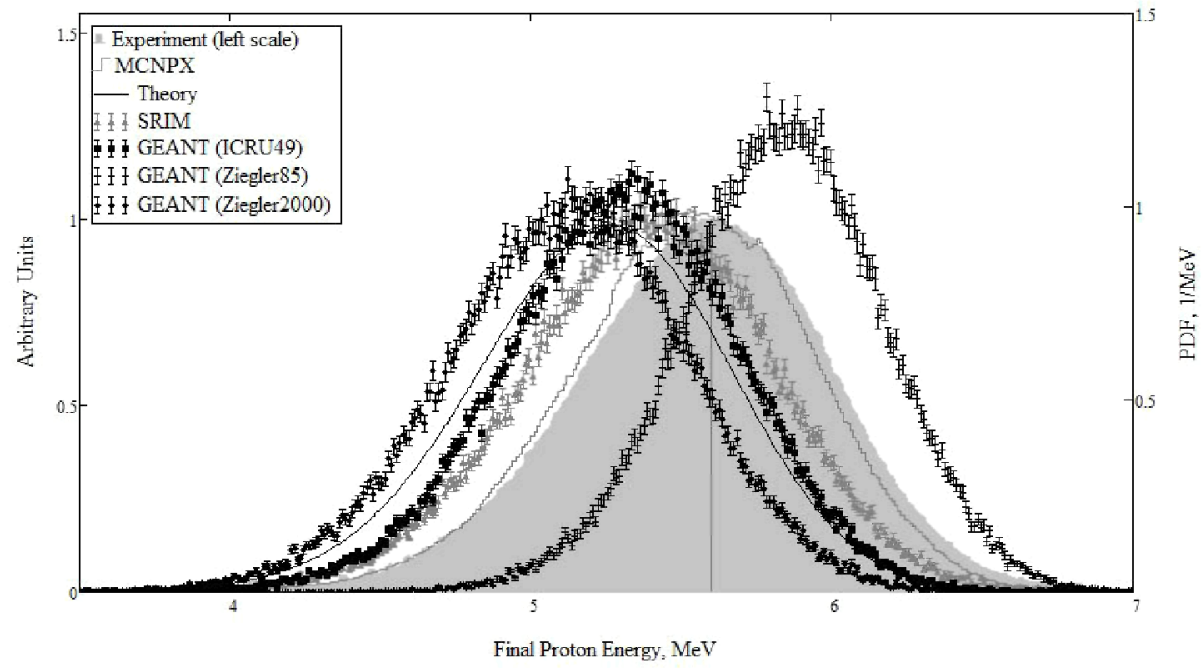

FIGURE 1. Simulated proton energy spectra for the $19.68 \mathrm{MeV}$ protons after $0.497 \mathrm{~g} / \mathrm{cm}^{2}$ of $\mathrm{Al}$ absorber.

Another interesting result is that NIST PSTAR always overestimates the proton energy loss: in the case of $0.497 \mathrm{~g} / \mathrm{cm}^{2}$ target the experimental spectrum has the mean energy $120 \mathrm{keV}$ greater. This obviously overcomes the energy resolution. For the SRIM and CSDA calculations the effect is even greater.

\section{CONCLUSIONS}

It was detected that in the case of thick Al target, the execution of GEANT4 code with various models from Low Energy Extension Pack leads to notable different spectra at the Bethe-Bloch domain. The results of present simulations show very clear some sort of proton energy loss overestimation in calculations performed at the base of NIST PSTAR, SRIM and CSDA models.

The comparison with only one set of experimental spectra was not helpful to make a definitive choice for the most adequate way to use GEANT4 for pCT simulations. Thus, we are planning to continue this work involving other experimental data. 


\section{ACKNOWLEDGMENTS}

The authors are greatly thankful to Dr. Reinhard Schulte from LLUMC (USA) for useful discussions. The work was supported by "Fundação Araucária" (Paraná State, Brazil), CAPES and CNPq.

\section{REFERENCES}

1. S. Agostinelli, J. Allison, K. Amako, et al., NIM, A506, 250-303, (2003).

2. J. Allison, K. Amako, J. Apostolakis, et al., IEEE Trans. Nucl. Sci., 53, 270-278, (2006).

3. H. F. W. Sadrozinski, V. Bashkirov, B. Keeney, et al., IEEE Trans. Nucl. Sci., 51, 3-9, (2004).

4. R. Schulte, V. Bashkirov, T. Li, et al. IEEE Trans. Nucl. Sci., 51, 866-872, (2004).

5. R. Schulte, M. C. L. Klock, V. Bashkirov, et al, Proc. SPIE, 5541, 153-164, (2004).

6. I. Evseev, M. C. L. Klock, S. A. Paschuk, et al, Brazilian Joumal of Physics, 34, 804-807, (2004)

7. R. Schulte, V. Bashkirov, M. C. L. Klock, et al, Med. Phys. 32, 1035-1046, (2005)

8. T. Li, Z. Liang, J. V. Singanallur, T. J. Satogata, et al., Med. Phys. 33, 699-706, (2006)

9. K. Amako, S. Guatelli, V. N. Ivanchenko, et al., IEEE Trans. Nucl. Sci., 52, 910-918, (2005).

10. J. T. de Assis, O. Yevseyeva, I. Evseev, et al., X Ray Spectrometry, 34, 481-492, (2005).

11.I. Evseev, J. T. de Assisa, O. Yevseyeva, et al., Proc. SPIE, 5745, 764-775, (2005).

12.O. Yevseyeva, J. T. de Assis, I. Evseev, et al., "The Scale-Invariance in pCT Method" in 2005 International Nuclear Atlantic Conference - INAC 2005, ABEN, 2005 (currently not assessable)

13 I. Evseev, J. T. de Assis, O. Yevseyeva, et al., "Comparison of Some Popular Monte Carlo Solutions for Proton Transportation within pCT Problem" in 2007 International Nuclear Atlantic Conference INAC 2007, ABEN, 2007, E05-1826 (http://www.inac2007.com.br/dvd/pdf dvd/E05 1826.pdf).

14.O. Yevseyeva, J. T. de Assis, I. Evseev, et al., "The comparison of Monte Carlo simulations within the pCT development" in XXX Reunião de Trabalho sobre Física Nuclear no Brasil, SBF, S.P. 200

15 J. F. Ziegler, NIM., B219-220, 1027-1036 (2004).

16.MCNPX User's Manual (Version 2.4.0), Los Alamos National Laboratory, LA-CP-02-408, USA, 2002.

17.C. Tschalär and H. D. Maccabee, Phys. Rev., B1, 2863-2869, (1970).

18. M. G. Payne, Phys. Rev., 185(2), 611-623, (1969).

19. G.A.P.Cirrone, "Medical application of the Geant 4 toolkit: Monte Carlo simulation of a proton therapy beam line". PhD Thesis in Physic University of Catania, 2003 (http://workgroup.lngs.infn.it/geant4lns/hadrontherapy example/hadrontherapy-geant4-advanced-example) 
Copyright of AIP Conference Proceedings is the property of American Institute of Physics and its content may not be copied or emailed to multiple sites or posted to a listserv without the copyright holder's express written permission. However, users may print, download, or email articles for individual use. 